\title{
Chirp Signal Transform and Its Properties
}

\author{
Mio Horai, ${ }^{1}$ Hideo Kobayashi, ${ }^{1}$ and Takashi G. Nitta $^{2}$ \\ ${ }^{1}$ Department of Electrical and Electronic Engineering, Faculty of Engineering Graduate school of Engineering, \\ Mie University, Kurimamachiyamachi, Tsu 514-8507, Japan \\ ${ }^{2}$ Department of Mathematics, Faculty of Education, Mie University, Kurimamachiyamachi, Tsu 514-8507, Japan
}

Correspondence should be addressed to Mio Horai; mio@com.elec.mie-u.ac.jp

Received 13 April 2014; Revised 19 July 2014; Accepted 19 July 2014; Published 20 October 2014

Academic Editor: Qiankun Song

Copyright (C) 2014 Mio Horai et al. This is an open access article distributed under the Creative Commons Attribution License, which permits unrestricted use, distribution, and reproduction in any medium, provided the original work is properly cited.

\begin{abstract}
The chirp signal exp $\left(i \pi(x-y)^{2}\right)$ is a typical example of CAZAC (constant amplitude zero autocorrelation) sequence. Using the chirp signals, the chirp $\mathrm{z}$ transform and the chirp-Fourier transform were defined in order to calculate the discrete Fourier transform. We define a transform directly from the chirp signals for an even or odd number $N$ and the continuous version. We study the fundamental properties of the transform and how it can be applied to recursion problems and differential equations. Furthermore, when $N$ is not prime and $N=M L$, we define a transform skipped $L$ and develop the theory for it.
\end{abstract}

\section{Introduction}

Chirp signal has been widely used in technology, for example, the radar system [1], as the linear FM pulse signal, the spectrum analyser as the sweep signal, the communications as the chirp modulation signal [2], and CAZAC sequence. Since the CAZAC sequences have constant amplitude and zero autocorrelation properties, they are now widely used in the fields of channel estimation and time and frequency synchronizations for OFDM (orthogonal frequency division multiplexing) and CDMA (code division multiple access) techniques which are employed as the standard transmission techniques in the wireless communications systems [3-5]. For the discrete Fourier transform theory, attached the chirp signal and Fourier transform, two kinds of transforms were already defined, namely, chirp z transform and chirp-Fourier transform. The first transform is (i) Fourier transform, (ii) product with the chirp signal, the second one is (i) product with the chirp signal (ii) Fourier transform [6]. These transforms were investigated mainly for the calculation of discrete Fourier transform (DFT).

In this paper, we define another transform directly treating the chirp signal, which is equal to (i) the product of the chirp signal, (ii) Fourier transform, and (iii) the product of the chirp signal, and which has a new meaning. Our motivation is totally different with the ones for the chirp $\mathrm{z}$ transform and the chirp-Fourier transform. We name it as chirp signal transform and consider the transform for an even or odd number $N$, continuous case, and skipped version in the case of $N=L M$. We calculate firstly some examples for the transform of discrete, continuous, and skipped versions. Secondly, we show the inverse transform for the transform elementary, not using usual Fourier transform theory. Thirdly we study the properties of the original chirp signal, which is fundamental for our theory. Finally, we apply the transform to the theory of recursion problems and ordinary differential and partial differential equations.

Chirp z transform and chirp-Fourier transform are based on the "orthogonality" of $\exp (i 2 \pi x y), y \in \mathbb{R}$; on the other hand, our chirp signal transform is founded on the orthogonality of $\exp \left(i \pi(x-y)^{2}\right), y \in \mathbb{R}$. These three kinds of transforms are changed to each other by the unitary transform $\exp \left(i \pi x^{2}\right)$ and $\exp \left(i \pi y^{2}\right)$; however, the represented meanings are completely different. The two transforms' $y$ is the meaning of the frequency and the remaining one's $y$ is the meaning of the position. The chirp signal $\exp (i \pi(x-$ $v t)^{2}$ ) is useful for the radar, because it can be generated by simple liner FM pulse which can increase the frequency bandwidth of pulse and accordingly improve the accuracy of range measurement. Moreover, the chirp signals (LFM or NLFM) have satisfied properties of ambiguity function for radar application. The chirp signal transform shows that any 
$L^{2}$-function is represented as a sum of such chirp signals with different central positions.

Furthermore, let $L$ and $\bar{L}$ be $(\partial / \partial x)+i 2 \pi x$ and $(\partial / \partial x)-$ $i 2 \pi x$. Since $[\bar{L}, L]=4 \pi i$, the set $\{L, \bar{L}\}$ generates the Lie algebra $s l(2, \mathbb{C})$. Additionally, $F L=i 2 \pi y F$ and $\overline{F L}=-i 2 \pi y \bar{F}$. It seems that the set $\{L, \bar{L}, F, \bar{F}\}$ has a good property for representation theory. It is very similar to the construction for the solution of harmonic oscillator in quantum mechanics by $L$ and $\bar{L}$. We expect to develop the algebraic theory for using the relationship of the differential operators $L, \bar{L}$ and the chirp transforms $F, \bar{F}$ in order to solve some differential equations.

\section{Definitions, Notations, and Examples}

In this section, we define chirp signal transform for discrete, continuous, and skipped versions.

For an even (resp., odd) $N$ and a constant number $a$ with $(a, N)=1$, we define chirp signal transform $F$ of the function over the set $\{0,1,2, \ldots, N-1\}$. Consider

$$
\begin{gathered}
F(f)(y)=\frac{1}{\sqrt{N}} \sum_{x=0}^{N-1} \exp \left(i \frac{a \pi}{N}(x-y)^{2}\right) f(x) \\
\left(\text { resp., } \frac{1}{\sqrt{N}} \sum_{x=0}^{N-1} \exp \left(\frac{i a \pi(x-y)(x+1-y)}{N}\right) f(x)\right) .
\end{gathered}
$$

When $N$ is odd especially, $F f(y)$ can be also defined by $(1 / \sqrt{N}) \sum_{x=0}^{N-1} \exp ((i 2 \pi(x-y)(x+1-y)) / N) f(x)$.

On the other hand, we also define chirp signal transform $F$ for continuous version, for $L^{2}(\mathbb{R})$,

$$
F(f)(y):=\int_{-\infty}^{\infty} \exp \left(i \pi(x-y)^{2}\right) f(x) d x .
$$

Since $\exp \left(i \pi(x-y)^{2}\right)=\exp \left(i \pi y^{2}\right) \cdot \exp (-i 2 \pi x y) \exp \left(i \pi x^{2}\right)$, the left hand side $F(f)(y)$ is equal to

$$
\exp \left(i \pi y^{2}\right)\left(\int_{-\infty}^{\infty} \exp (-i 2 \pi x y)\left(\exp \left(i \pi x^{2}\right) f(x)\right)\right) d x .
$$

We denote $\exp \left(i \pi x^{2}\right) f(x)$ to be $(U f)(x)$ and the Fourier transform as $\mathscr{F}$; then, $F$ is also written as $U \mathscr{F} U$. It is also the same for discrete cases. The transforms $U \mathscr{F}$ and $\mathscr{F} U$ are called as chirp z transform and chirp-Fourier transform [6].

Let $N$ be even and divided as $L \cdot M$. Then, we write

$$
e_{L, N}(x)= \begin{cases}\exp \left(i \frac{\pi}{L N} x^{2}\right), & \text { if } x \mid L \\ 0, & \text { if } x+L .\end{cases}
$$

We define chirp signal transform $F_{L, N}$ as $\left(F_{L, N} f\right)(y)=(1 /$ $\sqrt{M}) \sum_{x=0}^{N-1} f(x) \cdot e_{L, N}(x-y)$.

If $N$ is odd, both $c$ and $d$ are even or odd with $(c, N)=1$, and $N=L M$, then we define

$$
e_{L, N}(x)= \begin{cases}\exp \left(i \frac{\pi x}{L N}(c x+d)\right), & \text { if } x \mid L \\ 0, & \text { if } x+L .\end{cases}
$$

In the following, we calculate some examples for the above chirp signal transform $F$.

(i) For $a<b$, let $\chi_{[a, b]}$ be the step function $\chi_{[a, b]}(x)=$ $1(x \in[a, b])$ or $0(x \notin[a, b])$.

Then, the chirp signal transform of $\chi_{[a, b]}$ is the following:

$$
\begin{aligned}
\left(F \chi_{[a, b]}\right)(y) & =\int_{-\infty}^{\infty} \exp \left(i \pi(x-y)^{2}\right) \chi_{[a, b]}(x) d x \\
& =\int_{a}^{b} \exp \left(i \pi(x-y)^{2}\right) d x \\
& =\int_{a}^{b} \sum_{k=0}^{\infty} \frac{i \pi(x-y)^{2 k}}{k !} d x \\
& =\sum_{k=0}^{\infty}\left(\int_{a}^{b}(x-y)^{2 k} d x\right) \frac{(i \pi)^{k}}{k !} \\
& =\sum_{k=0}^{\infty}\left[\frac{1}{2 k+1}(x-y)^{2 k+1}\right]_{a}^{b} \frac{(i \pi)^{k}}{k !} \\
& =\sum_{k=0}^{\infty} \frac{(i \pi)^{k}}{k !(2 k+1)}\left\{(b-y)^{2 k+1}-(a-y)^{2 k+1}\right\} .
\end{aligned}
$$

(ii) We also calculate the chirp signal transform for the Gaussian distribution $\exp \left(-\pi x^{2}\right)$. Consider

$$
\begin{aligned}
\left(F\left(\exp \left(-\pi x^{2}\right)\right)\right)(y) \\
=\int_{-\infty}^{\infty} \exp \left(-\pi x^{2}\right) \exp \left(i \pi(x-y)^{2}\right) d x \\
=\int_{-\infty}^{\infty} \exp \left(-\pi\left(x^{2}-i(x-y)^{2}\right)\right) d x \\
=\exp \left(-\pi \frac{1-i}{2} y^{2}\right) \\
\quad \times\left(\int_{-\infty}^{\infty} \exp \left(-\pi(1-i)\left(x+\frac{i}{1-i} y\right)^{2}\right) d x\right) .
\end{aligned}
$$

Since $\exp \left(-\pi(1-i) z^{2}\right)$ is holomorphic and $\int_{-\infty}^{\infty} \exp (-\pi(1-$ $\left.i)(x+(i /(1-i)) y)^{2}\right) d x$ is constant up to $y$, we denote it as $c^{\prime}$.

The above is equal to $\exp \left(-\pi((1-i) / 2) y^{2}\right)\left(\int_{-\infty}^{\infty} \exp (-\pi(1-\right.$ i) $\left.x^{2}\right) d x$ ). Therefore,

$$
\left(F\left(\exp \left(-\pi x^{2}\right)\right)\right)(y)=c^{\prime} \exp \left(-\pi \frac{1-i}{2} y^{2}\right) .
$$

When $N$ is odd and $N=L M$, we calculate the skipped chirp transform of $f(x)=\exp (-2 \pi i(x y / N))$ at $y=0$ with 
respect to $\exp (i \pi(x(x+d) / L N)), d=L \alpha$, and $x=L m$. Now, $d$ is odd, and $\alpha$ is also odd. Consider

$$
\begin{aligned}
F_{L, N} & \left(\exp \left(-2 \pi i \frac{x k}{N}\right)\right)(0) \\
& =\sum_{x} \exp \left(i \pi \frac{L m(L m+L \alpha)}{L N}\right) \exp \left(-2 \pi i \frac{L m k}{N}\right) \\
& =\sum \exp \left(i \pi \frac{m^{2}+m \alpha-2 m k}{M}\right) \\
& =\sum_{m=0}^{M-1} \exp \left(\frac{i \pi(m-k)(m-k+\alpha)}{M}\right) \exp \left(\frac{i \pi k(\alpha-k)}{M}\right) \\
& =C \exp \left(\frac{\pi i k(\alpha-k)}{M}\right) .
\end{aligned}
$$

Finally, we explain briefly that the discrete chirp signal transform becomes the continuous one, when $N$ increases to the infinity.

Let $H$ be an even positive integer, and $1 / H$ is denoted by $\epsilon$. Then, we define $F_{H}$ for $f$ in $L^{2}(\mathbb{R})$,

$$
\begin{aligned}
\left(F_{H} f\right)(\epsilon m) & =\frac{1}{H} \sum_{n=-\left(H^{2} / 2\right)}^{H^{2} / 2} \exp \left(\frac{i \pi(n-m)^{2}}{H^{2}}\right) f(\epsilon n) \\
& =\frac{1}{H} \sum_{n=-\left(H^{2} / 2\right)}^{H^{2} / 2} \exp \left(i \pi(\epsilon n-\epsilon m)^{2}\right) f(\epsilon n) .
\end{aligned}
$$

Now,

$$
\begin{aligned}
(F f)(y) & =\int_{-\infty}^{\infty} \exp \left(i \pi(x-y)^{2}\right) f(x) d x \\
& =\lim _{L \rightarrow \infty} \int_{-(L / 2)}^{L / 2} \exp \left(i \pi(x-y)^{2}\right) f(x) d x \\
& =\lim _{L \rightarrow \infty} \lim _{H \rightarrow \infty} \frac{1}{H} \sum_{n=-(H / 2) L}^{(H / 2) L} \exp \left(i \pi(\epsilon n-y)^{2}\right) f(\epsilon n) .
\end{aligned}
$$

If the final $\lim _{L \rightarrow \infty} \lim _{H \rightarrow \infty}$ coincides to $\lim _{H \rightarrow \infty}$ under $L=H$, the above $F$ for the continuous case is the limit of the $F_{H}$ for the discrete case. When $H$ is odd, we can do the similar calculation. Furthermore, this correspondence can be represented as an infinitesimal lattice Fourier transform in nonstandard analysis [7-9].

\section{The Inverse Chirp Signal Transform $F^{-1}$}

In this section, we show the inverse transform of the chirp signal transform defined in the first section. Let $N$ be an integer, and let $K$ be the function space from $\{0,1, \ldots, N-1\}$ to the complex number field $\mathbb{C}$. For $f, g \in K$, the $L^{2}$ inner product is defined by

$$
(f, g)=\sum_{x=0}^{N-1} \overline{f(x)} g(x) .
$$

If $f(x-a), 0 \leq a \leq N-1$ are orthogonal to each other, then an arbitrary function $g \in K$ is represented as a linear combination of $\{f(x-a), 0 \leq a \leq N-1\}$. For example, in the case of even $N, f(x-a)=\exp \left(i a \pi\left((x-a)^{2} / N\right)\right)$ satisfies such property. Now,

$$
\begin{aligned}
& (\bar{F} F f)(z) \\
& =\frac{1}{N} \sum_{y=0}^{N-1}\left(\sum_{x=0}^{N-1} \exp \left(-i a \pi \frac{(y-z)^{2}}{N}\right)\right. \\
& \left.\quad \times \exp \left(i a \pi \frac{(x-y)^{2}}{N}\right) f(x)\right) \\
& =\frac{1}{N} \sum_{x=0}^{N-1}\left(\sum_{y=0}^{N-1} \exp \left(\frac{i a \pi(x+z-2 y)(x-z)}{N}\right)\right) f(x) .
\end{aligned}
$$

Since $\sum_{y=0}^{N-1} \exp ((-2 i a \pi(x-z)) / N) y$ is $N$ for $x=z$, or 0 for $x \neq z$, the above $(\bar{F} F f)(z)$ is equal to $f(z)$. Hence, $\bar{F} F=i d$, and $F^{-1}=\bar{F}$.

Similarly, when $N$ is odd, $f(x)=\exp (\operatorname{ia\pi }(x(x+c) / N))$ satisfies the same property; in fact, $(x-y)(x-y+c)-(x-$ $z)(x-z+c)=-2(y-z) x+(y-z)(y+z)-c(y-z)$.

Hence, $\left(F^{-1} f\right)(y)=(1 / \sqrt{N}) \sum_{x=0}^{N-1} \exp (-(i a \pi(x-y)(x-$ $y-c) / N)) f(x)$.

We remark that the suffix of $c$ is changed from positive to negative as $F$ to $F^{-1}$.

Next, we prove that $\bar{F} F=i d$ for the continuous version. We try to prove it directly.

Let $g_{t}(x)$ be $\exp \left(-\pi(t x)^{2}\right)$ for $t \in \mathbb{R}-\{0\}$. Then, the Fourier transform of $g_{t}$ is calculated as follows:

$$
\begin{aligned}
\left(\mathscr{F}\left(g_{t}\right)\right)(y)= & \int_{-\infty}^{\infty} \exp (-2 \pi i x y) \exp \left(-\pi(t x)^{2}\right) d x \\
= & \left(\int_{-\infty}^{\infty} \exp \left(-\pi\left(t x+i \frac{y}{t}\right)^{2}\right) d x\right) \\
& \times \exp \left(-\pi\left(\frac{y}{t}\right)^{2}\right),
\end{aligned}
$$

and we put $t x$ as $x^{\prime}$

$$
\begin{aligned}
& =\left(\int_{-\infty}^{\infty} \exp \left(-\pi\left(x^{\prime}+i \frac{y}{t}\right)^{2}\right) d x^{\prime}\right) \frac{1}{|t|} \exp \left(-\pi\left(\frac{y}{t}\right)^{2}\right), \\
& \text { as } \int_{-\infty}^{\infty} \exp \left(-\pi\left(x^{\prime}+i \frac{y}{t}\right)^{2}\right) d x^{\prime}=1, \\
& =\frac{1}{|t|} \exp \left(-\pi\left(\frac{y}{t}\right)^{2}\right) .
\end{aligned}
$$

Now, $\lim _{t \rightarrow \infty} g_{t}(x)=1$ and $\lim _{t \rightarrow 0}(1 /|t|) \exp \left(-\pi(y / t)^{2}\right)$ is equal to $\infty$ for $y=0$ and 0 for $y \neq 0$. We denote $(1 /|t|) \exp \left(-\pi(y / t)^{2}\right)$ to be $\delta_{t}(y)$; then, $\left(\mathscr{F}\left(g_{t}\right)\right)(y)$ is equal to $\delta_{t}(y)$. 
Theorem 1. $\bar{F} F f=f$ for $f \in L^{2}(\mathbb{R})$.

Proof. Consider

$$
\begin{aligned}
& \bar{F}(F(f))(z) \\
& =\int_{-\infty}^{\infty} \exp \left(-i \pi(y-z)^{2}\right)(F(f)(y)) d y \\
& =\lim _{t \rightarrow \infty} \int_{-\infty}^{\infty} g_{t}(y) \exp \left(-i \pi(y-z)^{2}\right)(F(f)(y)) d y \\
& =\lim _{t \rightarrow \infty} \int_{-\infty}^{\infty} g_{t}(y) \exp \left(-i \pi(y-z)^{2}\right) \\
& \quad \times\left(\int_{-\infty}^{\infty} \exp \left(i \pi(x-y)^{2}\right) f(x) d x\right) d y
\end{aligned}
$$

by Fubini's theorem,

$$
\begin{aligned}
& =\lim _{t \rightarrow \infty} \int_{-\infty}^{\infty} f(x)\left(\int_{-\infty}^{\infty} g_{t}(y) \exp \left(-i \pi(y-z)^{2}\right)\right. \\
& \left.\quad \times \exp \left(i \pi(x-y)^{2}\right) d y\right) d x \\
& =\lim _{t \rightarrow \infty} \int_{-\infty}^{\infty} f(x)\left(\int_{-\infty}^{\infty} g_{t}(y) \exp (-i 2 \pi(x-z) y) d y\right) \\
& =\lim _{t \rightarrow \infty} \int_{-\infty}^{\infty} f(x) \delta_{t}(x-z) \exp \left(i \pi\left(x^{2}-z^{2}\right)\right) d x \\
& =\lim _{t \rightarrow \infty} \int_{-\infty}^{\infty}\left(f(x) \cdot \exp \left(i \pi\left(x^{2}-z^{2}\right)\right)\right) \delta_{t}(x-z) d x \\
& =f(z) \cdot \exp (0)=f(z) .
\end{aligned}
$$

Finally, we show the inverse transform of the chirp signal transform for skipped version. When $N$ is even, we obtain the following.

Theorem 2. Functions $e_{L, N}(x-a), 0 \leq a \leq N-1$ are orthogonal to each other.

Proof. For $a, b$, if $a-b+L$; then, $\left(e_{L, N}(x-a), e_{L, N}(x-b)\right)=0$ by the definition. If $a-b \mid L$, then $a-b$ is written as $L \cdot \alpha$. Consider

$$
\begin{aligned}
\sum_{x=0}^{N-1} \overline{e_{L, N}} & (x-a) e_{L, N}(x-b) \\
& =\sum_{x=0}^{N-1} \overline{e_{L, N}}(x) e_{L, N}(x-(b-a))
\end{aligned}
$$

$$
\begin{aligned}
& =\sum_{x=0}^{N-1} \overline{e_{L, N}}(x) e_{L, N}(x-L \alpha) \\
& =\sum_{m=0}^{(N / L)-1} \overline{e_{L, N}}(L m) e_{L, N}(L m-L \alpha) \\
& =\sum_{m=0}^{(N / L)-1} \exp \left(-i \pi \frac{(L m)^{2}}{L N}\right) \exp \left(i \pi \frac{(L m-L \alpha)^{2}}{L N}\right) \\
& =\sum_{m=0}^{(N / L)-1} \exp \left(i \frac{\pi L^{2}}{L N}\left(\alpha^{2}-2 \alpha m\right)\right) \\
& =\exp \left(i \frac{\pi L}{N} \alpha^{2}\right) \sum_{m=0}^{(N / L)-1} \exp \left(-\frac{i 2 \pi}{N} L \cdot \alpha m\right) \\
& = \begin{cases}0, & \text { if } L \cdot \alpha+N \\
\exp \left(i \frac{\pi L}{N} \alpha^{2}\right) \cdot M, & \text { if } L \cdot \alpha \mid N .\end{cases}
\end{aligned}
$$

Hence, if $\alpha=0$, then $\left(e_{L, N}, e_{L, N}\right)=M$; otherwise, $\left(e_{L, N}(x-a), e_{L, N}(x-b)\right)=0$.

We assume that $N$ is odd. We obtain the following.

Theorem 3. Functions $e_{L, N}(x-a), 0 \leq a \leq N-1$ are orthogonal to each other.

Proof. We denote $d$ to be $d=L \beta$. For $a, b$, if $a-b+L$, then $\left(e_{L, N}(x-a), e_{L, N}(x-b)\right)=0$ by the definition. If $a-b \mid L$, then $a-b$ is written as $L \cdot \alpha$. Consider

$$
\begin{aligned}
\sum_{x=0}^{N-1} \overline{e_{L, N}} & (x-a) e_{L, N}(x-b) \\
= & \sum_{x=0}^{N-1} \overline{e_{L, N}}(x) e_{L, N}(x-(b-a)) \\
= & \sum_{x=0}^{N-1} \overline{e_{L, N}}(x) e_{L, N}(x-L \alpha) \\
= & \sum_{m=0}^{(N / L)-1} \overline{e_{L, N}}(L m) e_{L, N}(L m-L \alpha) \\
= & \sum_{m=0}^{(N / L)-1} \exp \left(-i \pi \frac{L m(c L m+L \beta)}{L N}\right) \\
= & \exp \left(-\frac{\pi i L}{L N} \alpha \beta\right) \sum_{m=0}^{(N / L)-1} \exp \left(-\frac{2 \pi i}{N} c L \alpha m\right)=0
\end{aligned}
$$

since $(c, N)=1$.

Hence, if $\alpha=0$, then $\left(e_{L, N}, e_{L, N}\right)=M$; otherwise, $\left(e_{L, N}(x-a), e_{L, N}(x-b)\right)=0$. 
The above theorem leads to the same property as the even case. Hence, for both cases, we obtain

$$
\left(F_{L, N}^{-1} f\right)(y)=\frac{1}{\sqrt{M}} \sum_{x=0}^{N-1} \bar{e}_{L, N}(y-x) f(x) .
$$

\section{Properties of the Chirp Signal}

In this section, we show some properties of the chirp signal $\exp \left(i \pi(x-y)^{2}\right)$ which is fundamental for our chirp signal transform.

Since $(\partial / \partial x)\left(\exp \left(i \pi(x-y)^{2}\right)\right)=2 \pi i(x-y) \exp \left(i \pi(x-y)^{2}\right)$, $((\partial / \partial x)-i 2 \pi x)\left(\exp \left(i \pi(x-y)^{2}\right)\right)=(-i 2 \pi y) \exp \left(i \pi(x-y)^{2}\right)$.

Now, we write the differential operators $(\partial / \partial x)+i 2 \pi x$, $(\partial / \partial x)-i 2 \pi x$ as $L, \bar{L}$. The differential operators $L$ and $\bar{L}$ satisfy the following properties:

$$
\begin{gathered}
\bar{L} L=\frac{d^{2}}{d x^{2}}+\left(4 \pi x^{2}+2 \pi i\right), \\
L \bar{L}=\frac{d^{2}}{d x^{2}}+\left(4 \pi x^{2}-2 \pi i\right), \\
{[\bar{L}, L]=\bar{L} L-L \bar{L}=4 \pi i .}
\end{gathered}
$$

Then, the chirp signal $\exp \left(i \pi(x-y)^{2}\right)$ is the eigen function of $\bar{L}$ with the eigen value $-i 2 \pi y$. Let $\Phi$ satisfy the equation $L \Phi=0$. Then, $\bar{L} L(\bar{L} \Phi)=\bar{L}(L \bar{L} \Phi)=\bar{L}(4 \pi i+\bar{L} L) \Phi=4 \pi i \bar{L} \Phi$. Similarly, $\bar{L} L\left(\bar{L}^{n} \Phi\right)=n \cdot 4 \pi i \bar{L}^{n} \Phi$; that is, $\bar{L}^{n} \Phi$ is the eigen function for $\bar{L} L$ with eigen value $n \cdot 4 \pi i$.

Let $N$ be an odd integer, and let $c, d$ be integers. Then, we define $f_{N, c, d}(x)$ as $\exp (-(i \pi x(c x+d) / N))$. We have the following.

Theorem 4. The function $f_{N, c, d}(x)$ is periodic iff both $c$ and $d$ are odd or even.

Proof. Consider

$$
\begin{aligned}
x(c x & +d)-(x+N)(c(x+N)+d) \\
& =-2 c x N+N(c N+d) \equiv 0 \quad(\bmod 2 N)
\end{aligned}
$$

iff $c N+d$ is even. Since $N$ is odd and both $c$ and $d$ are odd or even, $c N+d$ is always even.

Theorem 5. $f_{N, c, d}(x-a), 0 \leq a \leq N-1$ are orthogonal to each other, iff $a+N$.

Proof. Consider

$$
\begin{aligned}
& \sum_{x=0}^{N-1} \bar{f}(x) f(x+1) \\
&=\sum_{x=0}^{N-1} \exp \left(\frac{-i \pi x(a x+b)}{N}\right) \\
& \quad \times \exp \left(\frac{i \pi(x+1)(a(x+1)+b)}{N}\right)
\end{aligned}
$$

$$
\begin{gathered}
=\exp \left(\frac{\pi i(a+b)}{N}\right) \sum_{n=0}^{N-1} \exp \left(\frac{2 \pi i}{N} a n\right), \\
\exp (2 \pi i a / N) \neq 1 \quad \text { iff } a+N .
\end{gathered}
$$

Secondly, we calculate the Fourier transform for the chirp signal. Consider

$$
\begin{aligned}
& \frac{1}{\sqrt{N}} \sum_{x=0}^{N-1} \exp \left(-i \frac{\pi x^{2}}{N}\right) \exp \left(-i \frac{2 \pi x y}{N}\right) \\
& \quad=\frac{1}{\sqrt{N}} \sum_{x=0}^{N-1} \exp \left(-\frac{i \pi}{N}\left(x^{2}+2 x y\right)\right) \\
& \quad=\frac{1}{\sqrt{N}} \sum_{x=0}^{N-1} \exp \left(-i \frac{\pi}{N}\left((x+y)^{2}-y^{2}\right)\right) \\
& =\left(\frac{1}{\sqrt{N}} \sum_{x=0}^{N-1} \exp \left(-i \frac{\pi}{N}(x+y)^{2}\right)\right) \exp \left(i \frac{\pi y^{2}}{N}\right)
\end{aligned}
$$

Since $\exp \left(-i(\pi / N) x^{2}\right)$ is periodic,

$$
=\left(\frac{1}{\sqrt{N}} \sum_{x=0}^{N-1} \exp \left(-i \frac{\pi}{N} x^{2}\right)\right) \exp \left(i \frac{\pi y^{2}}{N}\right)
$$

we denote $(1 / \sqrt{N}) \sum_{x=0}^{N-1} \exp \left(-i(\pi / N) x^{2}\right)$ by $C$

$$
=C \exp \left(i \frac{\pi y^{2}}{N}\right)
$$

Now, since Fourier transform preserves the norm, $|C|=1$.

Hence, the Fourier transform for $\exp \left(-i\left(\pi x^{2} / N\right)\right)$ is equal to $C \exp \left(i\left(\pi y^{2} / N\right)\right)$.

Now, we assume $N$ is even, $L$ divides $N$, and $N=L M$. Consider

$$
f_{N, L}(x)= \begin{cases}\exp \left(\pi i \frac{x^{2}}{L N}\right), & x=\operatorname{Lm}(x \mid L) \\ 0, & x \neq \operatorname{Lm}(x+L) .\end{cases}
$$

We calculate the usual Fourier transform of $f_{N, L}(x)$. Consider

$$
\begin{aligned}
\left(\mathscr{F} f_{N, L}\right)(k) & \\
= & \frac{1}{\sqrt{N}} \sum_{x=0}^{N-1} f_{N, L}(x) \exp \left(-2 \pi i \frac{x k}{N}\right) \\
= & \frac{1}{\sqrt{N}} \sum_{m=0}^{M-1} \exp \left(\pi i \frac{(L m)^{2}}{L N}\right) \exp \left(\frac{-2 \pi i L m k}{N}\right)
\end{aligned}
$$




$$
\begin{aligned}
& =\frac{1}{\sqrt{N}} \sum_{m=0}^{M-1} \exp \left(\pi i \frac{m^{2}}{M}\right) \exp \left(\frac{-2 \pi i m k}{M}\right) \\
& =\frac{1}{\sqrt{N}} \sum_{m=0}^{M-1} \exp \left(\pi i \frac{(m-k)^{2}}{M}\right) \exp \left(-\pi i \frac{k^{2}}{M}\right) \\
& =\left(\frac{1}{\sqrt{N}} \sum_{m=0}^{M-1} \exp \left(\pi i \frac{m^{2}}{M}\right)\right) \exp \left(-\pi i \frac{k^{2}}{M}\right) .
\end{aligned}
$$

Next, we calculate the Fourier transform of $f_{N, L}(x-a)$. Consider

$$
\begin{aligned}
& \mathscr{F} f_{N, L}(x-a)(y)=\frac{1}{\sqrt{N}} \sum_{x} f(x-a) \exp \left(-2 \pi i \frac{x y}{N}\right), \\
& x^{\prime}:=x-a \\
& \quad=\frac{1}{\sqrt{N}} \sum_{x^{\prime}} f\left(x^{\prime}\right) \exp \left(-2 \pi i \frac{x^{\prime}+a}{N} y\right) \\
& \quad=\frac{1}{\sqrt{N}} \sum_{x^{\prime}} f\left(x^{\prime}\right) \exp \left(-2 \pi i \frac{x^{\prime} y}{N}\right) \exp \left(-2 \pi i \frac{a y}{N}\right) .
\end{aligned}
$$

Let $f(x, t)=\exp \left(i \pi(x-v t)^{2}\right)$. Then, $(\partial / \partial x) f=2 i \pi(x-$ $v t) f$ and $(\partial / \partial t) f=-2 i \pi(x-v t) v f$. Hence, $(\partial / \partial t) f=$ $-v(\partial / \partial x) f=-2 i \pi(x-v t) v f$. It is a chirp signal with central position $v t$, which is a wave of $x$ and $t$.

\section{Application for Recursion Formulas and Ordinary Differential Equations}

In this section, we develop some applications of the chirp signal transform.

(i) For a function $f$ from $\{0,1, \ldots, N-1\}$ to $\mathbb{C}$, we calculate the chirp signal transform of $f(x+1)$ with even $N$, $a=1$. Consider

$$
\begin{aligned}
(F f(x+1))(y) & \frac{1}{\sqrt{N}} \sum_{x=0}^{N-1} \exp \left(i \pi \frac{(x-y)^{2}}{N}\right) f(x+1) \\
= & \exp \left(i \pi \frac{2 y+1}{N}\right) \\
& \times\left\{\frac{1}{\sqrt{N}} \sum_{x^{\prime}=1}^{N} \exp \left(i \pi \frac{\left(x^{\prime}-y\right)^{2}}{N}\right)\right. \\
= & \exp \left(i \pi \frac{2 y+1}{N}\right)\left\{F\left(\exp \left(-i \frac{2 \pi x}{N}\right) f(x)\right)\right\}(y) .
\end{aligned}
$$

We apply this formula to the following equation for a constant $C$ and a given function $g(x)$ :

$$
f(x+1)+C \exp \left(-i \frac{2 \pi x}{N}\right) f(x)=g(x) .
$$

We transform this equation by the chirp signal transform using formula (30),

$$
\begin{aligned}
& \left(\exp \left(i \pi \frac{2 y+1}{N}\right)+C\right) F\left(\exp \left(-i \frac{2 \pi x}{N}\right) f(x)\right)(y) \\
& =F(g(x))(y) .
\end{aligned}
$$

We assume $C$ is not equal to $-\exp (i \pi((2 y+1) / N))$ for any $y \in\{0,1, \ldots, N-1\}$. For example, the absolute value of $C$ is not equal to 1 . Consider

$$
\begin{aligned}
& F\left(\exp \left(-i \frac{2 \pi x}{N}\right) f(x)\right)(y) \\
& =\frac{1}{\exp (i \pi((2 y+1) / N))+C} F(g(x))(y) ;
\end{aligned}
$$

hence, $f(x)=\exp (i(2 \pi x / N)) \cdot \bar{F}((1 /(\exp (i \pi((2 y+1) / N))+$ C) $F(g)(y))(x)$.

Similarly, we calculate the chirp signal transform of $\exp (i$ $(2 \pi(x+k) k / N)) f(x+k)$. Consider

$$
\begin{aligned}
& F\left(\exp \left(i \frac{2 \pi(x+k) k}{N}\right) f(x+k)\right)(y) \\
& =\frac{1}{\sqrt{N}} \sum_{x=0}^{N-1} \exp \left(i \pi \frac{(x-y)^{2}}{N}\right) \\
& \times \exp \left(i \frac{2 \pi(x+k) k}{N} f(x+k)\right), \\
& \left((x-y)^{2}+2(x+k) k=(x+k-y)^{2}+2 k y+k^{2}\right) \\
& =\exp \left(i \frac{\pi\left(2 k y+k^{2}\right)}{N}\right) \\
& \quad \times\left(\frac{1}{\sqrt{N}} \sum_{x=0}^{N-1} \exp \left(i \frac{\pi(x+k-y)^{2}}{N}\right) f(x+k)\right) \\
& =\exp \left(i \frac{\pi(2 y+k) k}{N}\right)(F f)(y) .
\end{aligned}
$$

Let $\left(T_{n} f\right)(x)$ be defined by $f(x+n)$. Then, $T_{n} \exp$ $\left(i \pi\left((x-y)^{2} / N\right)\right)$ is equal to $\exp \left(i \pi\left((x+n-y)^{2} / N\right)\right)$, that is, $\exp \left(i \pi\left((x-(y-n))^{2} / N\right)\right)$.

We consider the following equation:

$$
\sum_{k=0}^{m} C_{k} \exp \left(i \frac{2 \pi(x+k) k}{N}\right) T_{k} f(x)=g(x) .
$$

We put the chirp signal transform of it. Consider

$$
F\left(\sum_{k=0}^{m} C_{k} \exp \left(i \frac{2 \pi(x+k) k}{N}\right) T_{k} f(x)\right)=F(g(x)) .
$$


By equality (34),

$$
\left(\sum_{k=0}^{m} C_{k} \exp \left(i \frac{\pi(2 y+k) k}{N}\right)\right)(F f)(y)=F(g)(y) .
$$

If $\sum_{k=0}^{m} C_{k} \exp (i((\pi(2 y+k) k) / N)) \neq 0$, for $y \in\{0,1, \ldots, N-$ $1\}$, then

$(F f)(y)$

$$
=\frac{1}{\sum_{k=0}^{m} C_{k} \exp (i((\pi(2 y+k) k) / N))} F(g)(y) .
$$

Hence,

$$
f=F^{-1}\left(\frac{1}{\sum_{k=0}^{m} C_{k} \exp (i((\pi(2 y+k) k) / N))} F(g)(y)\right) .
$$

(ii) We put the assumption that $\lim _{x \rightarrow \pm \infty} f(x)=0$. Consider

$$
\begin{aligned}
\left(F f^{\prime}(x)\right)(y) & \\
= & \int_{-\infty}^{\infty} \exp \left(i \pi(x-y)^{2}\right) f^{\prime}(x) d x \\
= & {\left[f(x) \exp \left(i \pi(x-y)^{2}\right)\right]_{-\infty}^{\infty} } \\
& \quad-i 2 \pi \int_{-\infty}^{\infty}(x-y) f(x) \exp \left(i \pi(x-y)^{2}\right) d x
\end{aligned}
$$

by the assumption,

$$
\begin{aligned}
= & -i 2 \pi \int_{-\infty}^{\infty}(x f(x)) \exp \left(i \pi(x-y)^{2}\right) d x \\
& +i 2 \pi y \int_{-\infty}^{\infty} f(x) \exp \left(i \pi(x-y)^{2}\right) d x \\
= & -i 2 \pi F(x f(x))(y)+i 2 \pi y F(f(x))(y) .
\end{aligned}
$$

Hence, $F\left(f^{\prime}(x)\right)(y)=-i 2 \pi F(x f(x))(y)+i 2 \pi y F(f(x))(y)$ and

$$
F\left(\left(\frac{d}{d x}+i 2 \pi x\right) f\right)(y)=i 2 \pi y F(f)(y) .
$$

We write the differential operator $(d / d x)+i 2 \pi x$ as $L$; then, $F(L f)(y)=i 2 \pi y F(f)(y)$. We consider the following differential equation, for coefficients $C_{k}(0 \leq k \leq n)$ and a given function $g(x)$ :

$$
\sum_{k=0}^{n} C_{k} L^{k} f=g
$$

We apply the chirp signal transform:

$$
\begin{gathered}
F\left(\sum_{k=0}^{n} C_{k} L^{k} f\right)=F(g), \\
\sum_{k=0}^{n} C_{k} F\left(L^{k} f\right)=F(g), \\
\left(\sum_{k=0}^{n} C_{k}(i 2 \pi y)^{k}\right) F(f)=F(g) .
\end{gathered}
$$

If $\sum_{k=0}^{n} C_{k}(i 2 \pi y)^{k} \neq 0$ for arbitrary $y$, then $F(f)=\left(1 /\left(\sum_{k=0}^{n}\right.\right.$ $\left.\left.C_{k}(i 2 \pi y)^{k}\right)\right) F(g)$.

Therefore, $f=F^{-1}\left(\left(1 /\left(\sum_{k=0}^{n} C_{k}(i 2 \pi y)^{k}\right)\right) F(g)\right)$, formally.

For example, we consider the ordinary differential equation

$$
L f(x)+2 \pi f(x)=\delta(x),
$$

where $\delta(x)$ is the delta function. Since $F(\delta)(y)$ is equal to $\exp \left(i \pi y^{2}\right)$, the solution $f$ is just $F^{-1}((1 /$ $\left.(2 \pi i y+2 \pi)) \exp \left(i \pi y^{2}\right)\right)$, that is, $\left((1 / 2 \pi i) \int_{-\infty}^{\infty}((\exp \right.$ $(i 2 \pi x y)) /(y-i)) d y) \exp \left(-i \pi x^{2}\right)$. The integral $((1 / 2 \pi i)$ $\left.\int_{-\infty}^{\infty}((\exp (i 2 \pi x y)) /(y-i)) d y\right)$ is $\exp (-2 \pi x), 1 / 2,0$, in case $x>0, x=0, x<0$, respectively. Hence, $f(x)=\exp \left(-\pi\left(i x^{2}+2 x\right)\right), 1 / 2,0$ for $x>0, x=0, x<0$, respectively.

\section{Application for Partial Recursion Formulas and Differential Equations}

In this section, we consider the application for partial recursion formulas and differential equations.

(i) Let $f(x, t)$ be two variable function on $\{0,1, \ldots, N-$ $1\} \times \mathbb{R}$, and let

$$
\frac{\partial f}{\partial t}=\sum_{k=0}^{m} C_{k} \exp \left(i 2 \pi \frac{(x+k) k}{N}\right) T_{k} f(x) .
$$

Then

$$
\begin{array}{r}
F\left(\frac{\partial f}{\partial t}\right) \\
=F\left(\sum_{k=0}^{m} C_{k} \exp \left(i 2 \pi \frac{(x+k) k}{N}\right) T_{k} f(x)\right), \\
\frac{\partial(F f)}{\partial t}=\sum_{k=0}^{m} C_{k} \exp \left(i \pi \frac{(2 y+k) k}{N}\right) \cdot F f, \\
\log (F f)=\left(\sum_{k=0}^{m} C_{k} \exp \left(i \pi \frac{(2 y+k) k}{N}\right)\right) t+C, \\
F f=C \exp \left(t \sum_{k=0}^{m} C_{k} \exp \left(i \pi \frac{(2 y+k) k}{N}\right)\right), \\
f=C F^{-1}\left(\exp \left(t \sum_{k=0}^{m} C_{k} \exp \left(i \pi \frac{(2 y+k) k}{N}\right)\right)\right) .
\end{array}
$$

(ii) Let $f(x, t)$ be two variables function on $\mathbb{R} \times \mathbb{R}$. We write $L=(\partial / \partial x)+i 2 \pi x$.

Then, it is already shown as

$$
F(L f)(y)=i 2 \pi y F(f)(y) .
$$

Let $C_{k}(0 \leq k \leq n)$ be complex coefficients. Then, we consider the following partial differential equation:

$$
\frac{\partial}{\partial t} f=\sum_{k=0}^{n} C_{k} \cdot L^{k} f
$$


We put the chirp transform. Consider

$$
\begin{gathered}
F \frac{\partial}{\partial t} f=F\left(\sum_{k=0}^{n} C_{k} \cdot L^{k} f\right), \\
\frac{\partial}{\partial t}(F f)=\left(\sum_{k=0}^{n} C_{k}(i 2 \pi y)^{k}\right) F(f) .
\end{gathered}
$$

We divide the equation by $F f$,

$$
\frac{1}{F f} \frac{\partial}{\partial t}(F f)=\sum_{k=0}^{n} C_{k}(i 2 \pi y)^{k} .
$$

We put $\int d t$,

$$
l_{n}(F f)=\left(\sum_{k=0}^{n} C_{k}(i 2 \pi y)^{k}\right) t+C .
$$

We write $e^{c}=C$,

$$
F f=C \exp \left(\left(\sum_{k=0}^{n} C_{k}(i 2 \pi y)^{k}\right) t\right) .
$$

Hence,

$$
f=C F^{-1} \exp \left(\left(\sum_{k=0}^{n} C_{k}(i 2 \pi y)^{k}\right) t\right) .
$$

\section{Conclusions}

In this paper, we define the chirp signal transform using the chirp signal. Furthermore, we develop the theory of the chirp signal transform and apply it to the recursion problem, the ordinary differential equation, and the partial differential equation.

\section{Conflict of Interests}

The authors declare that they have no conflict of interests regarding the publication of this paper.

\section{Acknowledgment}

The authors would like to thank professor K. Hidano for his valuable suggestion and encouragement.

\section{References}

[1] P. Z. Peebles, Radar Principles, Wiley-Interscience Publication, 1998.

[2] B. Sklar, Digital Communications: Fundamentals and Applications, Prentice Hall, 2001.

[3] J. Meng and G. Kang, "A novel OFDM synchronization algorithm based on CAZAC sequence," in Proceedings of the International Conference on Computer Application and System Modeling (ICCASM '10), pp. 634-637, October 2010.
[4] Y. Chunlin, L. Shaoqian, and T. Youxi, "New frequency o set estimation method for OFDM systems by using CAZAC sequence," Journal of Electrics \& Information Technology, pp. 139-412, 2006.

[5] A. Kebo, I. Konstantinidis, J. J. Benedetto, M. R. Dellomo, and J. M. Sieracki, "Ambiguity and sidelobe behavior of CAZAC coded waveforms," in Proceedings of the IEEE Radar Conference, pp. 99-103, Boston, Mass, USA, April 2007.

[6] X. Xia, "Discrete chirp-Fourier transform and its application to chirp rate estimation," IEEE Transactions on Signal Processing, vol. 48, no. 11, pp. 3122-3133, 2000.

[7] T. Nitta and Y. Péraire, "Divergent Fourier analysis using degrees of observability," Nonlinear Analysis: Theory, Methods \& Applications, vol. 71, no. 12, pp. e2462-e2468, 2009.

[8] T. Nitta, "A complexified path integral for a system of harmonic oscillators," Nonlinear Analysis: Theory, Methods \& Applications, vol. 71, no. 12, pp. e2469-e2473, 2009.

[9] T. Nitta, "Complexification of the propagator for the harmonic oscillator," in Topics in Contemporary Differential Geometry, Complex Analysis and Mathematical Physics, pp. 261-268, World Science Publisher, Hackensack, NJ, USA, 2007. 


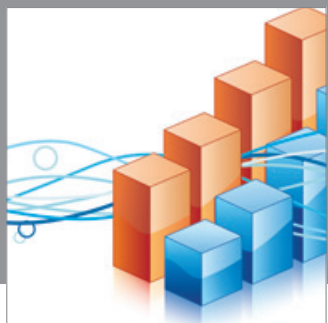

Advances in

Operations Research

mansans

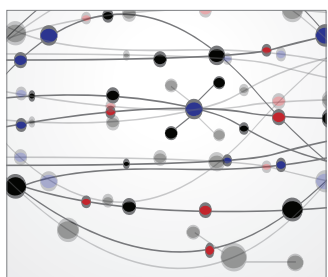

The Scientific World Journal
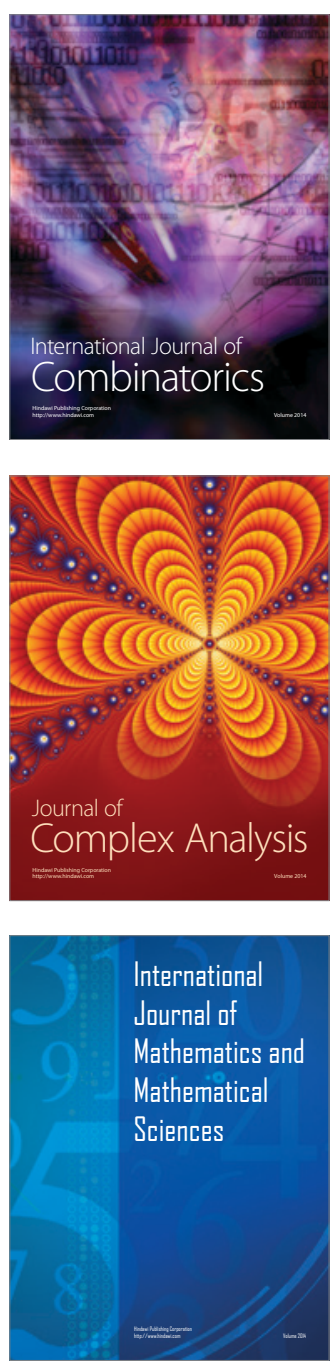
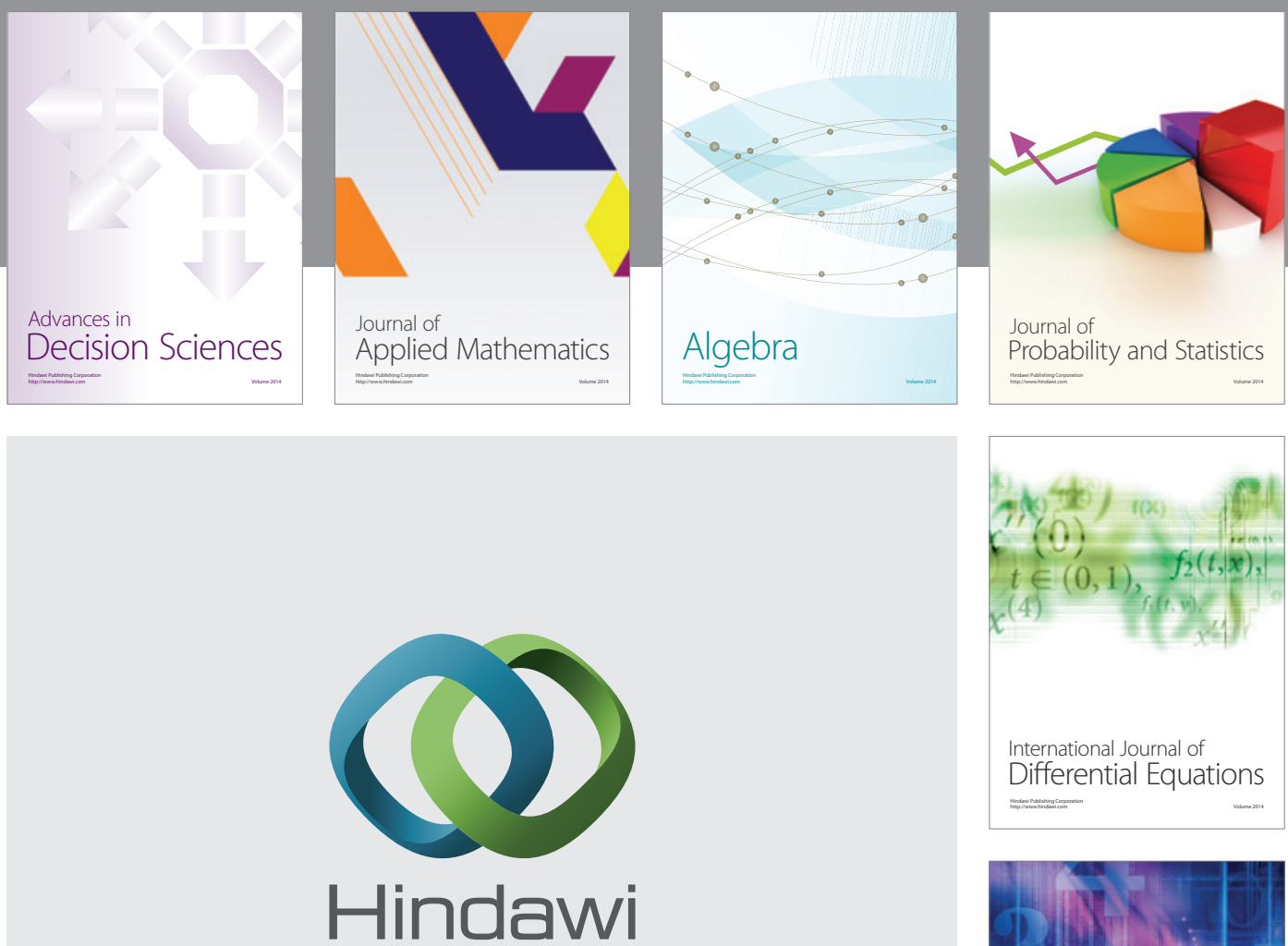

Submit your manuscripts at http://www.hindawi.com
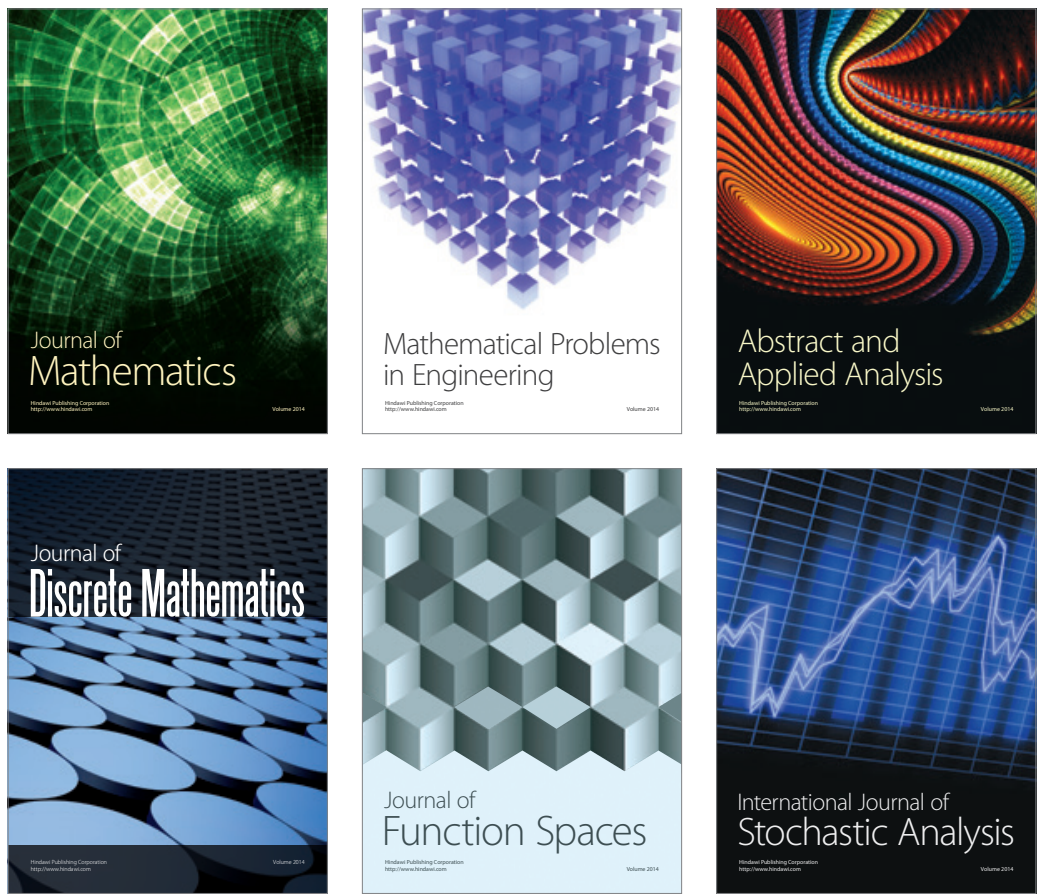

Journal of

Function Spaces

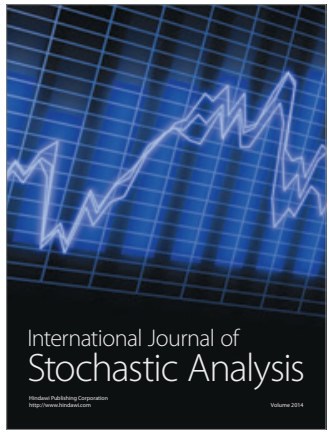

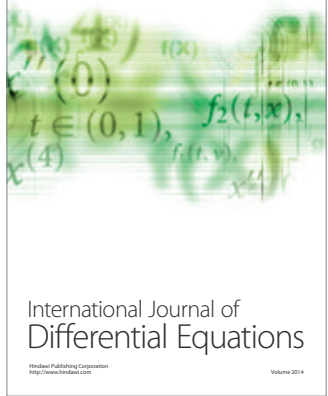
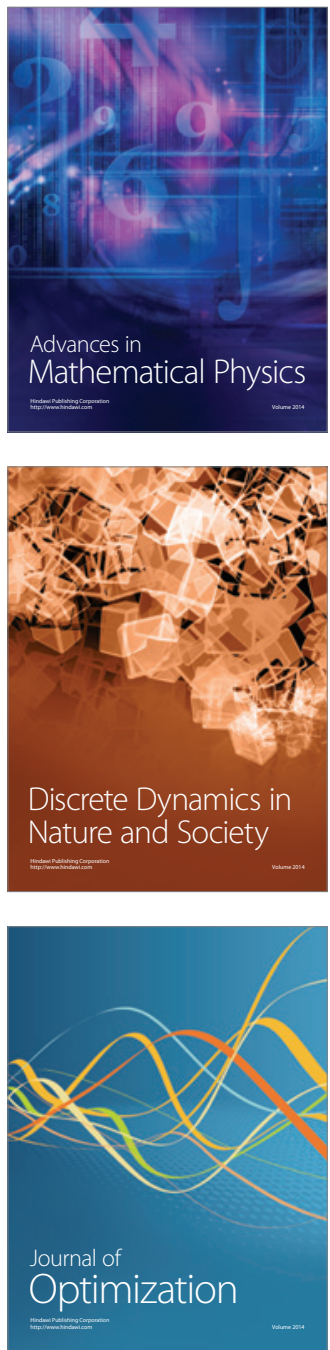